\title{
A validated nomogram integrating hematological indicators to predict response to neoadjuvant therapy in esophageal squamous cell carcinoma patients
}

\author{
Sichao Wang ${ }^{1 \#}$, Zihao Zhou ${ }^{1 \#}$, Dan Tian ${ }^{1 \#}$, Shujie Huang ${ }^{1,2}$, Ce Wang ${ }^{3}$, Zhen Gao ${ }^{1,4}$, Xiaosong Ben ${ }^{1}$, \\ Jiming Tang ${ }^{1}$, Liang Xie ${ }^{1}$, Haiyu Zhou ${ }^{1}$, Dongkun Zhang ${ }^{1}$, Ruiqing Shi ${ }^{1}$, Cheng Deng ${ }^{1}$, Weitao Zhuang ${ }^{1,2}$, \\ Yu Ding ${ }^{1,4}$, Guibin Qiao ${ }^{1}$ \\ ${ }^{1}$ Department of Thoracic Surgery, Guangdong Provincial People's Hospital, Guangdong Academy of Medical Sciences, Guangzhou, China; ${ }^{2}$ Shantou \\ University Medical College, Shantou, China; ${ }^{3}$ Department of Radiology, Guangdong Provincial People's Hospital, Guangdong Academy of Medical \\ Sciences, Guangzhou, China; ${ }^{4}$ The Second Clinical Medical School, Southern Medical University, Guangzhou, China \\ Contributions: (I) Conception and design: S Wang, Z Zhou, D Tian, G Qiao; (II) Administrative support: G Qiao; (III) Provision of study materials \\ or patients: S Wang, Z Zhou, D Tian; (IV) Collection and assembly of data: All authors; (V) Data analysis and interpretation: All authors; (VI) \\ Manuscript writing: All authors; (VII) Final approval of manuscript: All authors. \\ \#These authors contributed equally to this work. \\ Correspondence to: Guibin Qiao, MD, PhD. Department of Thoracic Surgery, Guangdong Provincial People's Hospital, Guangdong Academy of \\ Medical Sciences, 106 Zhongshan Second Road, Guangzhou 510080, China. Email: guibinqiao@126.com.
}

Background: The prognoses for advanced Esophageal squamous cell cancer (ESCC) was very poor. Neoadjuvant therapy was shown to improve overall survival of ESCC patients. However, there is still no effective indicator to predict the efficacy of neoadjuvant therapy. The present study intended to investigate the correlation between hematological parameters and the efficacy of neoadjuvant therapy so as to provide a reference for the prediction of cancer response to neoadjuvant therapy.

Methods: This study included 197 ESCC patients in our center from January 2010 to December 2018. Response evaluation criteria in solid tumors (RECIST) criteria were used for the treatment evaluation. The results of univariate and multivariate logistic regression analysis were used to select independent factors for construction of the prediction model. The concordance index (C-index), receiver operating characteristic (ROC) curve, and calibration curve were used to evaluate the robustness of the model, while the bootstrap method was used for internal validation.

Results: Among the 197 included ESCC patients, 94 patients achieved partial remission, 80 patients were in stable condition, and 23 patients had disease progression, 123 of whom underwent surgery. The comparisons of the dynamic hematological test results before and after treatment show that pre-PLT, pre$\mathrm{MONO} \%$, post- $\mathrm{Hb}, \triangle \mathrm{WBC}$, and the option of undergoing neoadjuvant chemoradiation were the potential predictors for the effectiveness of neoadjuvant therapy. The model in which the C-index was 0.803 (95\% confidence interval: $0.742-0.864$ ) showed good prediction performance, and still reach a C-index of 0.764 when internally validated.

Conclusions: For the neoadjuvant treatment of ESCC, hematological indexes are closely related to the efficacy of neoadjuvant therapy. The nomogram can be used to easily predict the efficacy of neoadjuvant therapy in patients.

Keywords: Esophageal squamous cell carcinoma (ESCC); neoadjuvant therapy; hematology test; nomogram; response prediction

Submitted Mar 05, 2021. Accepted for publication Apr 13, 2021.

doi: $10.21037 / \mathrm{atm}-21-1628$

View this article at: http://dx.doi.org/10.21037/atm-21-1628 


\section{Introduction}

Esophageal cancer is the seventh most common malignant tumor in the world and has the sixth highest cancerrelated mortality (1). The annual incidence and mortality of esophageal cancer in China account for more than half of the total in esophageal cancer in the world (2), with the main pathological type be esophageal squamous cell carcinoma (ESCC). Owing to subtle symptoms of esophageal cancer at the early stage, most patients have already reached the locally advanced stage at the time of diagnosis, and their prognoses are usually unsatisfactory. Recently, neoadjuvant therapy followed by surgery has become the standard therapy for patients with locally advanced esophageal cancer. Pathological remission, especially pathological complete response (pCR) after neoadjuvant therapy, is closely associated with the favorable survival of esophageal cancer patients (3). Neoadjuvant therapy can effectively control local lesions, increase R0 resection rate, and improve patients' prognosis. However, there is currently no effective indicator to predict the efficacy of neoadjuvant therapy, and related laboratory research has mainly focused on the tumor molecular level $(4,5)$, such as tumor metabolomics, gene expression pathways, and protein expression levels. These studies ignore the complexity of a patient as an organic whole, and the results of such basic research cannot be easily transformed into economical clinical evaluation methods.

To address the, the present study focused on the patient's hematological test results. Recent studies have found that differential levels of hematological parameters such as white blood cells (WBCs), red blood cells (RBCs) and platelets (PLT_ are significantly associated with esophageal cancer in patients' prognosis; for instance, poor prognosis has been shown to be caused by lymphopenia (6), and tumor progression was found to be promoted by neutrophils (NE) (7). Numerous studies have explored the influences of NE to lymphocyte (LM) ratio (NLR), PLT to LM ratio (PLR), and other indicators on the prognosis of advanced cancer, and it is generally believed that higher NLR and PLR lead to worse prognosis (8). Some esophageal cancer patients are likely to undergo surgery. However, there are currently few studies on the relationship between the prognosis of neoadjuvant therapy for ESCC and hematological tests, and the sample sizes the related studies are usually small. The aim of the present study was thus to provide a reference for predicting the efficacy of neoadjuvant therapy by analyzing the association between the results of hematological testing and the efficacy of neoadjuvant therapy. We further developed a nomogram based on the readily available indicators, including blood test results and baseline characteristics of patients, to predict ESCC patients' response to neoadjuvant therapy. To the best of our knowledge, this study includes by far the largest retrospective sample size to be used to investigate neoadjuvant efficacy and hematological prognosis in the field of ESCC. This innovation that obtains relevant factors, by analyzing the dynamic changes of the patient's blood routine results during the neoadjuvant treatment process is also closer to clinical applications in the real world. We present the following article in accordance with the TRIPOD reporting checklist (available at http://dx.doi. org/10.21037/atm-21-1628).

\section{Methods}

\section{Patient selection}

This study included patients who received and completed neoadjuvant therapy (neoadjuvant chemoradiation or neoadjuvant chemotherapy) at the Department of Thoracic Surgery, Guangdong Provincial People's Hospital between January 2010 and December 2018. The patients included in the study were clearly diagnosed as ESCC by preoperative endoscopic biopsy, and the patients' tumors were defined as locally advanced by preoperative imaging. In addition, the included patients had complete blood routine test results and imaging data examinations completed before and after neoadjuvant therapy, and had no fever or inflammation. The study was approved by an independent ethical committee review board at Guangdong Provincial People's Hospital (No. GDREC2020253H) and written informed consent was obtained from all patients. All procedures performed in this study involving human participants were in accordance with the Declaration of Helsinki (as revised in 2013).

\section{Treatment plan}

All patients underwent neoadjuvant chemotherapy or concurrent neoadjuvant chemoradiation, and response evaluation criteria in solid tumors RECIST v.1.1 (9) criteria were used to evaluate the treatment effect. Treatment evaluation was confirmed jointly by an experienced radiologist and an experienced thoracic surgeon. This study used the eighth edition of the American Joint Committee on Cancer (AJCC) esophageal cancer staging system to 


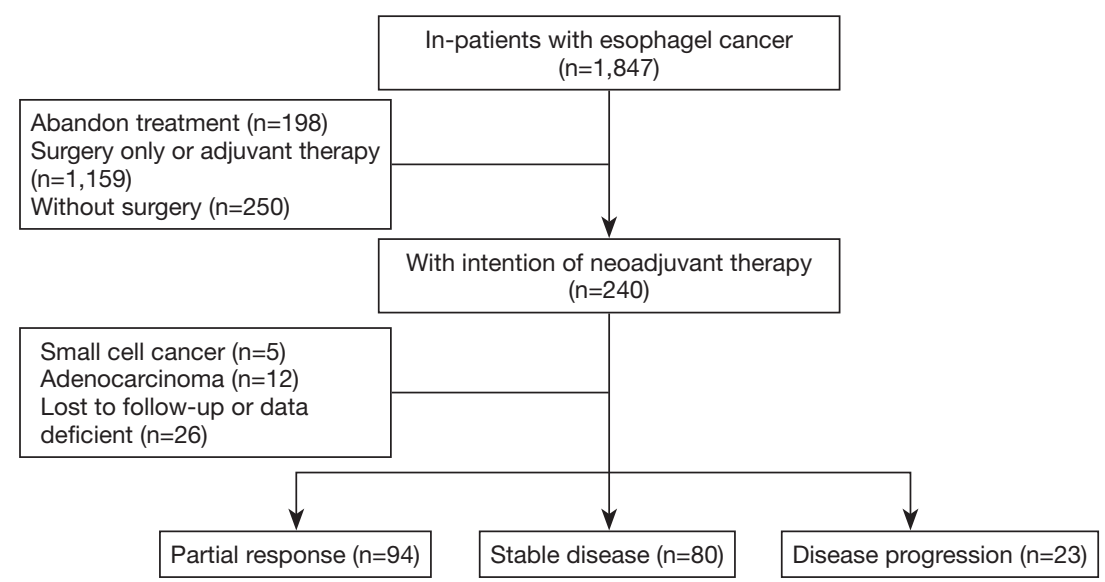

Figure 1 Patient selection criteria for inclusion for statistical analysis.

restage the patients' clinical stage (10). Tumor differentiation was divided into a G1 high-differentiation group, a G2 medium-differentiation group, and a G3 low-differentiation group. The patients evaluated as partial response $(\mathrm{PR})$ were recommended to undergo surgical treatment and those who were evaluated as stable disease (SD) but had strong desire to receive surgery were also provided surgical treatment. Surgical methods included Sweet, Ivor Lewis, and McKeown. The remaining patients who did not undergo surgery continued to received chemotherapy or concurrent chemoradiation.

\section{Histopatbological examination}

In order to determine the pathological type and differentiation status of the tumor, patients were provided gastroscopic biopsy before surgery; for those who were able to undergo surgery, their surgically removed specimens underwent paraffin pathological sectioning, so that the type of residual tumor, depth of invasion, differentiation condition, lymph node status, and the efficacy of neoadjuvant therapy could be comprehensively evaluated.

\section{Data and statistical metbods}

Grouping of patients was performed according to criteria of effectiveness and ineffectiveness. The patients with $\mathrm{PR}$ were included in the effective group, while patients with SD or progressive disease (PD) were included in the ineffective group. All patients' blood routine data before neoadjuvant therapy, after neoadjuvant therapy, and before imaging reassessment were collected; the collected data included WBC, LM, NE, monocytes (MONO), eosinophils (EO), basophils (BASO), RBC, hemoglobin (Hb), PLT, albumin (ALB), alanine aminotransferase (ALT); PLT to $\mathrm{LM}$ ratio (PLR), NE to $\mathrm{LM}$ ratio (NLR), and NE to WBC-NE ratio (dNLR). Student's $t$-test or Mann-Whitney test was used for continuous variable data; Pearson's chisquare $\left(\chi^{2}\right)$ and Kruskal-Wallis one-way analysis of variance (ANOVA) were used for unclassified categorical variable data; logistics analysis was used for correlation analysis. A $P$ value $<0.05$ was considered statistically significant. The above data methods were analyzed by SPSS software v 23.0 (IBM Corp.) and $\mathrm{R}$ version 4.0.3 (Foundation for Statistical Computing). The results of the multifactor analysis and potential predictors were used to build a predictive model for neoadjuvant therapy response (11). A calibration curve was plotted to assess the nomogram. In order to evaluate the predictive performance of the nomogram, the area under the curve (AUC) and concordance index (C-index) were measured. Bootstrapping validation of the nomogram (10,000 bootstrap resamples) was used to calculate the relative corrected $\mathrm{C}$-index (12).

\section{Results}

\section{Patient characteristics}

According to the screening criteria, a total of 197 ESCC patients with neoadjuvant treatment intention were selected at the Department of Thoracic Surgery, Guangdong Provincial People's Hospital from January 2010 to December 2018. The screening process is shown in Figure 1. As shown in Table 1, the mean age of included patients was 
Page 4 of 12

Table 1 Characteristics of the 197 patients analyzed

\begin{tabular}{lc}
\hline Characteristic & Patients, $\mathrm{n}(\%)(\mathrm{n}=197)$ \\
\hline Mean age, yrs (SD) & $59(7.9)$ \\
Gender & \\
Male & $165(83.8 \%)$ \\
Female & $32(16.2 \%)$ \\
Smoker & $106(53.8 \%)$ \\
Drinker & $65(32.9 \%)$ \\
Tumor location & \\
Upper & $27(13.7 \%)$ \\
Mid & $98(49.7 \%)$ \\
Lower & $72(36.5 \%)$ \\
Clinical stage & \\
II & $28(14.2 \%)$ \\
III & $156(79.1 \%)$ \\
IVA & $13(6.5 \%)$ \\
Neoadjuvant therapy type & \\
Neoadjuvant chemotherapy & \\
Neoadjuvant chemoradiotherapy & $54(27.4 \%)$ \\
PR & \\
\hline PD & \\
PR & \\
Pense evaluation & $(41.7 \%)$ \\
\hline
\end{tabular}

PR, partial response; SD, stable disease; PD, progressive disease.

59 , and the age at the time of diagnosis ranged between 50 and 70 years. Most of the patients were male (165 people, $83.8 \%$ ). Half of the patients had a smoking habit and had not quit, and about one-third of the patients drank alcohol. The neoadjuvant therapeutic regimen received by the patients were mainly chemotherapy alone $(143,72.6 \%)$. The tumor condition is shown in Table 2. The tumor location of the included patients was mainly in the middle segment (98, $49.7 \%)$ and the lower segment $(72,36.5 \%)$. Nearly half of the patients $(94,47 \%)$ showed PR to neoadjuvant therapy. The pCR indicates that no tumor cell residue can be seen under the microscope (13), and there were 12 patients with pCR, accounting for about $6.1 \%$ of the total sample. The clinical stage of included patients was mainly stage III (156, $80.4 \%)$.

\section{Clinical characteristics analysis}

From the comparative analysis between the effective group and ineffective group, we found that there was no statistical difference in the age of patients $(\mathrm{P}=0.704)$, but that there was a statistical difference in gender $(\mathrm{P}=0.01)$. The smoking habit $(\mathrm{P}=0.002)$ and drinking habit $(\mathrm{P}=0.006)$ of patients between groups were statistically significant. The type of neoadjuvant therapy also showed a significant difference in neoadjuvant efficacy (simple chemotherapy $v s$. concurrent chemoradiation $=41.3 \%$ vs. $64.8 \% ; \mathrm{P}=0.003$ ). This study further analyzed the location and differentiation degree of tumor, the results show that the location of tumor had a correlation with neoadjuvant efficacy to a certain extent $(-0.157 ; \mathrm{P}=0.028)$. Moreover, the results of single factor regression analysis indicated that the neoadjuvant efficacy in patients seemed to be worse if their tumor was located in a lower position [lower esophageal cancer: odds ratio (OR) $0.374,95 \%$ confidence interval (CI): $0.150-1.933, \mathrm{P}=0.035$ ]. However, there was no statistical between the effective group and ineffective group in the tumor differentiation degree $(\mathrm{P}=0.465)$ or the clinical stage at diagnosis $(\mathrm{P}=0.167)$.

\section{Blood routine analysis}

The difference of blood routine data before neoadjuvant therapy and imaging reassessment between the 2 groups of patients was also analyzed. Results revealed that prePLT, pre-PLR, pre-MONO, pre-MONO\%, post-Hb, post-WBC, post-NE, post-PLT, post-MONO, post-ALB, $\triangle \mathrm{Hb}, \triangle \mathrm{WBC}, \triangle \mathrm{NE}, \triangle \mathrm{NE} \%, \triangle \mathrm{NLR}$, and $\triangle \mathrm{dNLR}$ had significant differences between the 2 groups. As shown in Table 2, pre-PLT (effective group vs. ineffective group: $245 \pm 80$ vs. $296 \pm 98, \mathrm{P}<0.001$ ), pre-PLR (effective group $v s$. ineffective group: $140 \pm 57$ vs. $164 \pm 69, \mathrm{P}=0.009)$, preMONO (effective group $v s$. ineffective group: $0.59 \pm 0.27 v s$. $0.67 \pm 0.28, \mathrm{P}=0.028$ ), and pre-MONO\% (effective group vs. ineffective group: $7.9 \% \pm 3.2 \%$ vs. $9.0 \% \pm 3.3 \%, \mathrm{P}=0.019$ ) were all related to the efficacy of neoadjuvant therapy; meanwhile, post-WBC (effective group $v s$. ineffective group: $5.38 \pm 1.89$ vs. $6.48 \pm 2.59, \mathrm{P}=0.001$ ), post-NE (effective group $v s$. ineffective group: $3.16 \pm 1.52$ vs. $3.95 \pm 2.02, \mathrm{P}=0.002$ ), post-MONO (effective group $v s$. ineffective group: $0.54 \pm 0.22 v s .0 .69 \pm 0.37, \mathrm{P}=0.001$ ), post-PLT (effective group vs. ineffective group: $222 \pm 89$ vs. $253 \pm 89, \mathrm{P}=0.017$ ), and post-HB (effective group $v s$. ineffective group: $120 \pm 14$ vs. $114 \pm 16, \mathrm{P}=0.005$ ) were found to be statistically significant indicators after neoadjuvant treatment. Further 
Table 2 Correlations of blood indicators between the effective group and ineffective group

\begin{tabular}{|c|c|c|c|}
\hline Characteristic & $\begin{array}{l}\text { Effective group, } \\
\qquad P R, n=94\end{array}$ & $\begin{array}{l}\text { Ineffective group, } \\
S D+P D, n=103\end{array}$ & $P$ value \\
\hline \multicolumn{4}{|l|}{ Pre-NAT } \\
\hline Pre-RBC & $4.44 \pm 0.45$ & $4.46 \pm 0.64$ & 0.815 \\
\hline Pre-Hb & $133.87 \pm 12.20$ & $132.33 \pm 14.97$ & 0.432 \\
\hline Pre-WBC & $7.44 \pm 1.87$ & $7.56 \pm 1.96$ & 0.681 \\
\hline Pre-NE & $4.72 \pm 1.55$ & $4.68 \pm 1.57$ & 0.847 \\
\hline Pre-NE\% & $62.66 \% \pm 8.76 \%$ & $61.15 \% \pm 8.13 \%$ & 0.210 \\
\hline Pre-LM & $1.91 \pm 0.66$ & $1.95 \pm 0.55$ & 0.664 \\
\hline Pre-LM\% & $26.36 \% \pm 8.23 \%$ & $26.53 \% \pm 6.93 \%$ & 0.876 \\
\hline Pre-BASO & $0.024 \pm 0.018$ & $0.025 \pm 0.024$ & 0.688 \\
\hline Pre-BASO\% & $0.32 \% \pm 0.25 \%$ & $0.34 \% \pm 0.30 \%$ & 0.887 \\
\hline Pre-EO & $0.194 \pm 0.144$ & $0.221 \pm 0.271$ & 0.389 \\
\hline Pre-EO\% & $2.65 \% \pm 1.99 \%$ & $2.87 \% \pm 2.89 \%$ & 0.550 \\
\hline Pre-MONO & $0.587 \pm 0.269$ & $0.674 \pm 0.279$ & 0.028 \\
\hline Pre-MONO\% & $7.93 \% \pm 3.22 \%$ & $9.03 \% \pm 3.34 \%$ & 0.019 \\
\hline Pre-PLT & $244.85 \pm 79.82$ & $296.02 \pm 97.63$ & $<0.001$ \\
\hline Pre-PLR & $139.76 \pm 57.30$ & $163.56 \pm 68.53$ & 0.009 \\
\hline Pre-NLR & $2.80 \pm 1.54$ & $2.55 \pm 1.03$ & 0.552 \\
\hline Pre-dNLR & $1.84 \pm 0.71$ & $1.69 \pm 0.60$ & 0.128 \\
\hline Pre-ALB & $37.71 \pm 4.04$ & $37.12 \pm 3.91$ & 0.299 \\
\hline Pre-ALT & $17.98 \pm 12.91$ & $17.14 \pm 11.01$ & 0.623 \\
\hline \multicolumn{4}{|l|}{ Post-NAT } \\
\hline Post-RBC & $3.90 \pm 0.47$ & $3.89 \pm 0.73$ & 0.545 \\
\hline Post-Hb & $120.05 \pm 12.58$ & $113.93 \pm 16.46$ & 0.005 \\
\hline Post-WBC & $5.39 \pm 1.89$ & $6.48 \pm 2.59$ & 0.001 \\
\hline Post-NE & $3.16 \pm 1.52$ & $3.95 \pm 2.02$ & 0.002 \\
\hline Post-NE\% & $56.48 \% \pm 12.39 \%$ & $59.33 \% \pm 12.23 \%$ & 0.105 \\
\hline Post-LM & $1.51 \% \pm 0.67$ & $1.65 \% \pm 0.71$ & 0.152 \\
\hline Post-LM\% & $29.29 \% \pm 11.16 \%$ & $26.79 \% \pm 10.81 \%$ & 0.113 \\
\hline Post-BASO & $0.022 \pm 0.021$ & $0.024 \pm 0.025$ & 0.575 \\
\hline Post-BASO\% & $0.40 \% \pm 0.30 \%$ & $0.39 \% \pm 0.35 \%$ & 0.880 \\
\hline Post-EO & $0.167 \pm 0.203$ & $0.174 \pm 0.220$ & 0.804 \\
\hline Post-EO\% & $3.13 \% \pm 3.39 \%$ & $2.70 \% \pm 3.32 \%$ & 0.358 \\
\hline Post-MONO & $0.537 \pm 0.217$ & $0.686 \pm 0.372$ & 0.004 \\
\hline Post-MONO\% & $10.63 \% \pm 4.35 \%$ & $10.82 \% \pm 3.70 \%$ & 0.735 \\
\hline
\end{tabular}

Table 2 (continued)
Table 2 (continued)

\begin{tabular}{|c|c|c|c|}
\hline Characteristic & $\begin{array}{l}\text { Effective group, } \\
\qquad \mathrm{PR}, \mathrm{n}=94\end{array}$ & $\begin{array}{l}\text { Ineffective group, } \\
S D+P D, n=103\end{array}$ & $P$ value \\
\hline Post-PLT & $222.43 \pm 89.11$ & $253.16 \pm 90.00$ & 0.017 \\
\hline Post-PLR & $175.16 \pm 101.60$ & $202.54 \pm 181.23$ & 0.198 \\
\hline Post-NLR & $2.42 \pm 1.47$ & $3.08 \pm 2.80$ & 0.184 \\
\hline Post-dNLR & $1.50 \pm 0.78$ & $1.80 \pm 1.32$ & 0.059 \\
\hline Post-ALB & $36.94 \pm 4.03$ & $35.22 \pm 4.83$ & 0.008 \\
\hline Post-ALT & $25.74 \pm 22.54$ & $21.28 \pm 16.27$ & 0.11 \\
\hline \multicolumn{4}{|l|}{ Variation value } \\
\hline$\triangle \mathrm{RBC}$ & $-0.54 \pm 0.43$ & $-0.57 \pm 0.51$ & 0.584 \\
\hline$\triangle \mathrm{Hb}$ & $-13.82 \pm 13.19$ & $-19.39 \pm 15.11$ & 0.025 \\
\hline$\triangle W B C$ & $-2.06 \pm 1.97$ & $-1.07 \pm 2.59$ & 0.003 \\
\hline$\triangle \mathrm{NE}$ & $-1.57 \pm 1.71$ & $-0.73 \pm 2.07$ & 0.002 \\
\hline$\triangle \mathrm{NE} \%$ & $-6.18 \% \pm 12.01 \%$ & $-1.82 \% \pm 11.84 \%$ & 0.011 \\
\hline$\triangle \mathrm{LM}$ & $-0.40 \pm 0.53$ & $-0.30 \pm 0.72$ & 0.139 \\
\hline$\triangle \mathrm{LM} \%$ & $2.93 \% \pm 10.43 \%$ & $0.27 \% \pm 10.53 \%$ & 0.076 \\
\hline$\triangle \mathrm{BASO}$ & $-0.001 \pm 0.023$ & $-0.001 \pm 0.028$ & 0.859 \\
\hline$\triangle \mathrm{BASO} \%$ & $0.08 \% \pm 0.33 \%$ & $0.05 \% \pm 0.41 \%$ & 0.622 \\
\hline$\triangle \mathrm{EO}$ & $-0.270 \pm 0.201$ & $-0.046 \pm 0.259$ & 0.558 \\
\hline$\triangle \mathrm{EO} \%$ & $0.48 \% \pm 3.44 \%$ & $-0.17 \% \pm 3.35 \%$ & 0.178 \\
\hline$\triangle \mathrm{MONO}$ & $-0.051 \pm 0.263$ & $0.012 \pm 0.373$ & 0.178 \\
\hline$\triangle \mathrm{MONO} \%$ & $2.70 \% \pm 4.43 \%$ & $1.79 \% \pm 4.14 \%$ & 0.138 \\
\hline$\triangle P L T$ & $-22.42 \pm 83.56$ & $-42.86 \pm 96.12$ & 0.114 \\
\hline$\triangle P L R$ & $35.40 \pm 91.67$ & $38.98 \pm 164.09$ & 0.195 \\
\hline$\triangle \mathrm{NLR}$ & $-0.38 \pm 1.73$ & $0.53 \pm 2.70$ & 0.006 \\
\hline$\triangle \mathrm{dNLR}$ & $-0.34 \pm .81$ & $0.10 \pm 1.28$ & 0.005 \\
\hline$\triangle \mathrm{ALB}$ & $-0.77 \pm 4.42$ & $-1.90 \pm 5.15$ & 0.101 \\
\hline$\triangle \mathrm{ALT}$ & $7.74 \pm 21.21$ & $4.13 \pm 17.70$ & 0.196 \\
\hline
\end{tabular}

$\mathrm{PR}$, partial response; $\mathrm{SD}$, stable disease; $\mathrm{PD}$, progressive disease; NAT, neoadjuvant therapy; RBC, red blood cell; $\mathrm{Hb}$, hemoglobin; WBC, white blood cell; NE, neutrophil; LM, Iymphocyte; BASO, basophil; EO, eosinophil; MONO, monocyte; PLT, platelet; PLR, PLT to LM ratio; NLR, NE to LE ratio; dNLR, NE to WBC-NE ratio; ALB, albumin; ALT, alanine aminotransferase. 
comparative analysis of these 2 occasions of blood routine were conducted; the results showed that the higher reduction degree of $\triangle \mathrm{WBC}$ (effective group $v$ s. ineffective group: $-2.06 \pm 1.97 v s .-1.07 \pm 2.59, \mathrm{P}=0.003$ ), $\triangle \mathrm{NE}$ (effective group vs. ineffective group: $-1.57 \pm 1.71$ vs. $-0.73 \pm 2.07$, $\mathrm{P}=0.002$ ), $\triangle \mathrm{NE} \%$ (effective group $v s$. ineffective group: $-6.2 \% \pm 12.0 \%$ vs. $-1.8 \% \pm 11.8 \%, \mathrm{P}=0.011$ ), $\triangle \mathrm{NLR}$ (effective group $v s$. ineffective group: $-0.38 \pm 1.73 v s$. $0.52 \pm 2.70$, $\mathrm{P}=0.006$ ), and $\triangle \mathrm{dNLR}$ (effective group $v s$. ineffective group: $-0.34 \pm 0.81$ vs. $0.10 \pm 1.28, \mathrm{P}=0.005)$ after treatment was significantly associated with the efficacy of neoadjuvant therapy. Related studies have discovered NLR and PLR are related to the prognosis of ESCC patients (14), and similar results were produced in this study. We also discovered that the decrease of $\mathrm{Hb}$ was more pronounced in the ineffective group (effective group $v s$. ineffective group: $-13.82 \pm 13.19$ vs. $-18.39 \pm 15.11, \mathrm{P}=0.025)$.

\section{Neoadjuvant effect predictor}

When performing predictive factor analysis, this study included all indicators with statistical differences. In binary logistics regression analysis, the effect of neoadjuvant therapy was taken as the dependent variable, and the following independent variables were included: pre-PLT, pre-PLR, pre-MONO, pre-MONO\%, post-HB, postWBC, post-NE, post-PLT, post-MONO, post-ALB, $\triangle \mathrm{Hb}$, $\triangle \mathrm{WBC}, \triangle \mathrm{NE}, \triangle \mathrm{NE} \%, \triangle \mathrm{NLR}, \triangle \mathrm{dNLR}$, gender, smoking habit, drinking habit, tumor location, and treatment program. As shown in Table 3, the final analysis results revealed that low pre-PLT (OR 0.991, 95\% CI: $0.985-$ 0.997, $\mathrm{P}=0.003$ ), low pre-MONO\% (OR 0.810, 95\% CI: 0.674-0.974, $\mathrm{P}=0.025$ ), high post-HB (OR 1.036, 95\% CI: 1.009-1.064, $\mathrm{P}=0.008), \triangle \mathrm{WBC}(\mathrm{OR} 0.404,95 \%$ CI: 0.189 $0.865, \mathrm{P}=0.020), \triangle \mathrm{Hb}(\mathrm{OR} 1.023,95 \% \mathrm{CI}: 1.003-1.044$, $\mathrm{P}=0.027$ ), and neoadjuvant chemoradiation (OR 2.782, $95 \%$ CI: $1.400-5.526, \mathrm{P}=0.003)$ were all potential predictors of the neoadjuvant effect. Overall, lower PLT and lower $\mathrm{MONO} \%$ before neoadjuvant therapy, higher $\mathrm{Hb}$ after neoadjuvant treatment, and greater reduction of $\mathrm{WBC}$ and lesser decrease of $\mathrm{Hb}$ during treatment seemed to indicate a better efficacy of neoadjuvant therapy. In addition, neoadjuvant chemoradiation could lead to better efficacy of neoadjuvant therapy.

\section{Development of an individualized prediction model}

First, the model was established based on the relevant characteristics of the patients before the initial treatment, which is called the baseline nomogram (Figure 2A). The calibration curves of the baseline nomogram in the cohort exhibited good consistency (Figure 2B). The receiver operating characteristic (ROC) of the prediction model is presented in Figure $2 C$, and the AUC was 0.723 . The C-index of the baseline nomogram was 0.723 (95\% CI: $0.652-0.793$ ) and was confirmed to be 0.690 by bootstrap validation. This indicated that the predictive performance of the baseline nomogram was good.

We then combined the neoadjuvant therapy type and related variation characteristics during the treatment process to build the model and obtain the response nomogram (Figure $3 A$ ). Compared to the baseline nomogram, there was a more consistent calibration curve (Figure 3B), larger AUC area (Figure 3C), and better C-index (0.803), and bootstrapvalidated $\mathrm{C}$-index $(0.7638)$ in the response nomogram.

\section{Discussion}

Surgery-based multimodality therapeutic approaches remain an important strategy for esophageal cancer patients in obtaining better prognosis. However, most of these patients have reached the locally advanced stage at the time of initial diagnosis, so their prognoses are usually poor. Effective neoadjuvant therapy can allow for the opportunity of surgery and better long-term benefits to those patients in the locally advanced stages. Nevertheless, there is currently no effective index to predict the efficacy of neoadjuvant therapy. Considering that the therapeutic effect is related to individual differences and the immune level of patients (15), this study attempted to use the blood routine test, which is one of the most basic clinical testing methods, to identify relevant predictors of neoadjuvant efficacy by means of exploring the correlation between blood routine indicators and the effect of neoadjuvant therapy. Through comparing the blood routine data before and after neoadjuvant treatment, we found that pre-PLT, pre-PLR, pre-MONO, pre-MONO\%, post-Hb, post-WBC, post-NE, post-PLT, post- $\mathrm{MONO}$, post-ALB, $\triangle \mathrm{Hb}, \triangle \mathrm{WBC}, \triangle \mathrm{NE}, \triangle \mathrm{NE} \%$, $\triangle \mathrm{NLR}$, and $\triangle \mathrm{dNLR}$ were associated with neoadjuvant efficacy. The patient's gender, smoking habit, drinking habit, tumor location, and therapy options were also significantly associated with the efficacy of neoadjuvant treatment. These indicators were included in the binary logistics regression analysis, and the results show that low pre-PLT, low pre$\mathrm{MONO} \%$, high post-Hb, a high reduction of $\triangle \mathrm{WBC}$ degree, a low reduction of $\triangle \mathrm{HB}$, and patient's option of 
Table 3 Univariate and multivariate analyses

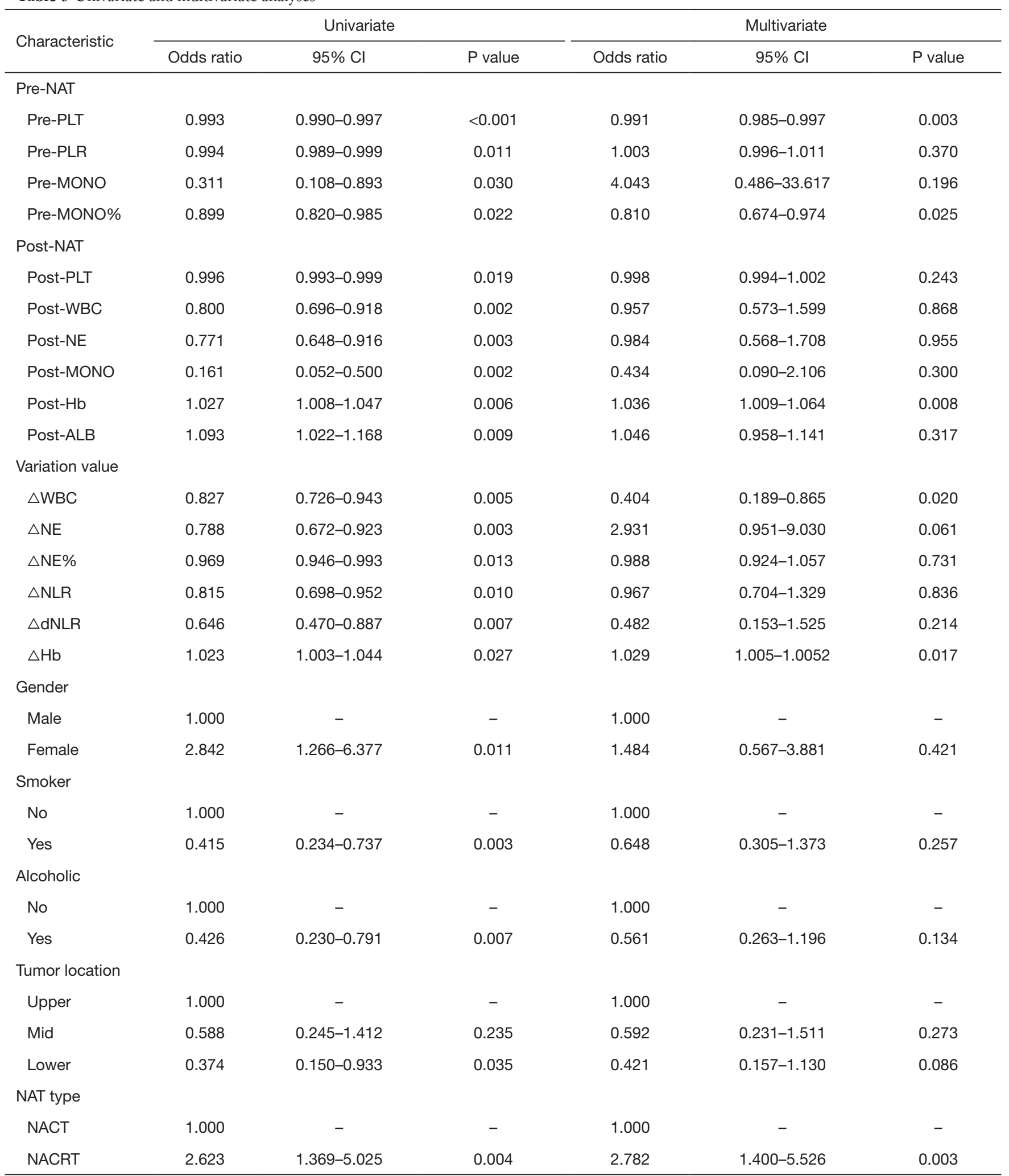

NAT, neoadjuvant therapy; PLT, platelet; PLR, PLT to LM ratio; MONO, monocyte; WBC, white blood cell; NE, neutrophil; Hb, hemoglobin; ALB, albumin; NLR, NE to LE ratio; dNLR, NE to WBC-NE ratio. 

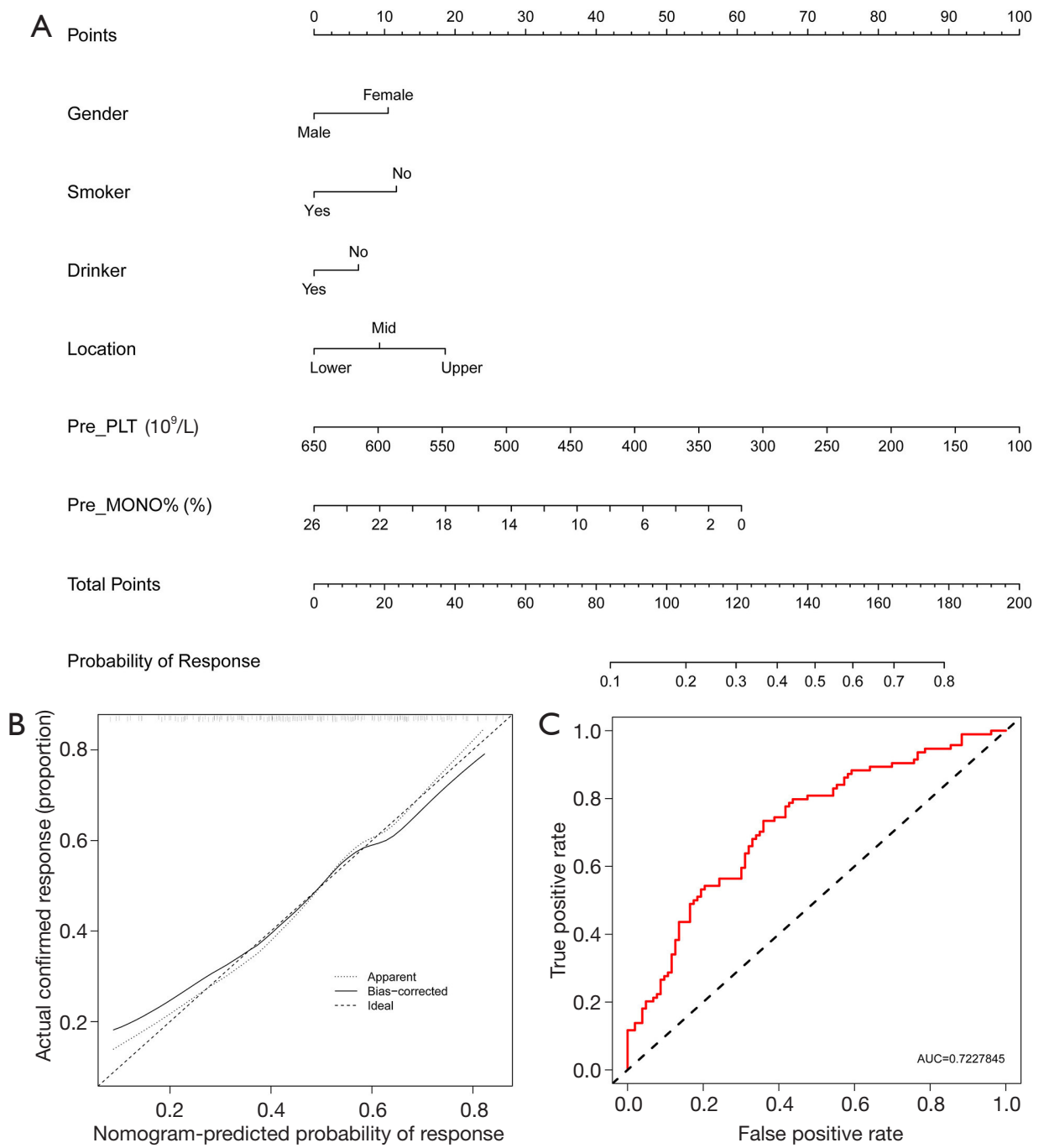

Figure 2 Baseline nomogram was established based on the relevant characteristics of the patients before the initial treatment and calibration curve exhibited good consistency. (A) Baseline nomogram, (B) calibration curve of baseline nomogram, and (C) receiver operating characteristic (ROC) curve of the basic model.

radiochemotherapy were the predictors of neoadjuvant therapy efficacy.

The immune system plays an important role in resisting tumor, and various types of blood cells play different parts in tumor development and invasion resistance. For example, platelets actively participate in tumor's extravasation, growth, metastasis, neovascularization, immune escape, and other processes, while lymphocytes, such as T cells, B cells, and natural killer (NK) cells, play a role in attacking cancer. In the 1970s, Klein discovered that tumor-infiltrating T cells exhibit antitumor activity (16). Since then, whether the molecular mechanism of the interactions between immune cells and tumor cells on the microscopic level can be translated into macroscopic clinical data and provide references for clinical treatment has been thoroughly discussed.

As an important component in hemostasis, platelets also play an essential role in the development of tumors. Cancer is often considered to be a chronic trauma, which can promote platelet activation. By stimulating the proliferation of vascular endothelial cells and smooth muscle cells, activated platelets can promote tumor neovascularization so as to ensure the energy supply to tumor tissues and help tumor cells adhere to the walls of blood vessels, 

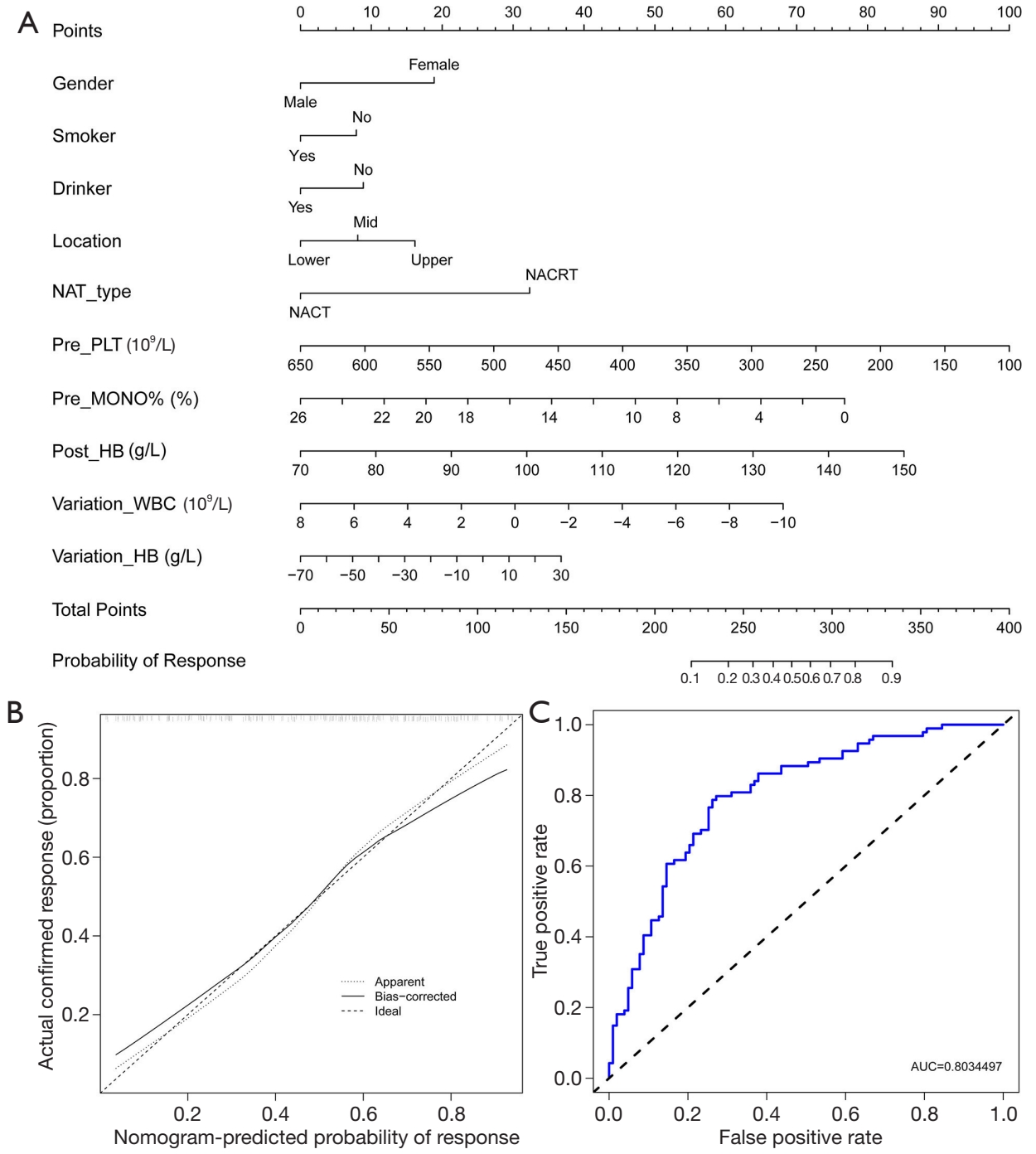

Figure 3 Response nomogram was built by combining the neoadjuvant therapy type and related variation characteristics during the treatment process. (A) Response nomogram, (B) calibration curve of response nomogram, and (C) receiver operating characteristic (ROC) curve of the model.

promoting the formation of tumor thrombosis and deep vein thrombosis. Then, platelets attach to the tumor cells that enter the circulatory system and further strengthen the adhesion, extravasation, and metastatic abilities of tumor cells (17). Macroscopically, the high platelet baseline level is a negative predictor of cancer patient's prognosis, which is consistent with the results of this study. This phenomenon can be observed in breast cancer (18), rectal cancer (19), and other tumors. Similar mechanisms also appear with MONOs. In the tumor microenvironment, inflammatory MONOs promote tumor cell extravasation, while tumorassociated macrophages derived from peripheral blood
MONOs can produce large amounts of anti-inflammatory factors to promote tumor development and simultaneously suppress acquired immunity, accelerating tumor growth and tumor angiogenesis and subsequently inducing metastasis and invasion. Furthermore, previous studies have shown that the number of peripheral blood mononuclear cells can reflect the status and level of tumor-associated macrophages in patients $(20,21)$. For breast cancer patients, the mononuclear cell count in peripheral blood is a poor prognostic factor for survival (22), which is line with the findings of this study. The number and percentage of MONOs in the ineffective group were higher than those in 
the effective group, and the percentage of MONOs was a poor predictor of the efficacy of neoadjuvant therapy (OR 0.810, 95\% CI: 0.674-0.974, $\mathrm{P}=0.025$ ).

Neoadjuvant therapy induces inhibitory effects on various lines of peripheral blood cells. In this study, the $\mathrm{Hb}$ levels in effective group and ineffective group both declined after treatment, and the degree of reduction in the ineffective group was higher (effective group $v s$. ineffective group: $-13.82 \pm 13.19$ vs. $-18.39 \pm 15.11, \mathrm{P}=0.025)$. Furthermore, the results of statistical analysis showed that the $\mathrm{Hb}$ level in the effective group was higher than that in the ineffective group after neoadjuvant treatment (effective group $v s$. ineffective group: $120 \pm 14$ vs. $114 \pm 16, \mathrm{P}=0.005$ ). The results of correlation analysis also demonstrated that the variations of $\mathrm{Hb}$ level between the effective group and ineffective group were statistically different (OR 1.023, 95\% CI: $1.003-1.044, \mathrm{P}=0.027)$. That is, either a lower decline of $\mathrm{Hb}$ or a higher $\mathrm{Hb}$ level after neoadjuvant therapy is an effective predictor of neoadjuvant therapy. A previous metaanalysis on rectal cancer found that preoperative anemia was significantly associated with long-term overall survival (OS) and disease-free survival (DFS) reduction in patients with rectal cancer (23), and the same phenomenon was also observed in studies of bladder cancer (24) and ovarian cancer (25), which suggests that relatively low $\mathrm{Hb}$ levels are associated with poor prognosis in cancer patients. Low $\mathrm{Hb}$ level indicates that the patient is relatively hypoxic, and this hypoxic environment provides a suitable environment for tumor cells to grow and subsequently develop resistance to neoadjuvant therapy. Additionally, a higher $\mathrm{Hb}$ level implies that patients have better nutritional status and general conditions so that they are more likely to be benefit from the impact of neoadjuvant therapy on the body. Contrary to the $\mathrm{Hb}$ conditions, the level of WBCs also decreased after neoadjuvant therapy in both groups, but the degree of reduction was higher in the effective group (effective group $v s$. ineffective group: $-2.06 \pm 1.97$ vs. $-1.07 \pm 2.59, \mathrm{P}=0.003$ ). In addition, the variation of $\mathrm{WBC}$ level in the effective group was significantly different from that in the ineffective group (OR 0.404, 95\% CI: 0.189-0.865, P=0.020). This study also found that the decline of NEs was the main contributor to the reduction of WBCs (effective group $v s$. ineffective group: $-1.57 \pm 1.71$ vs. $-0.73 \pm 2.07, \mathrm{P}=0.002$ ), suggesting that a greater decrease of NEs during treatment may reflect a better effectiveness of neoadjuvant therapy. Between the effective and ineffective groups, there were statistical differences in the variations of WBCs, NEs count, $\mathrm{NE}$ ratio, and the variation of NLR and dNLR. NEs play a positive role in the occurrence, development, and metastasis of tumors, and tumors promote the generation of NEs by upregulating various cytokines and chemokines (26). Most of the related research thus far has shown that NLR is a risk factor for poor prognosis, with these studies supporting the notion that NEs promote rather than inhibit cancer progression. Additionally, dNLR has also been widely investigated as a predictor in the field of tumor immunotherapy (27).

The results of logistics analysis indicated that the type of neoadjuvant therapy was most relevant to the efficacy of neoadjuvant therapy. Neoadjuvant radiochemotherapy was significantly more effective than neoadjuvant chemotherapy and induced a higher pCR rate, which is consistent with the findings of previous meta-analyses (28). Nevertheless, whether neoadjuvant chemotherapy and neoadjuvant radiochemotherapy can confer long-term benefits in patients is still unknown.

As a predictive tool, the nomogram is widely used in the prediction of oncology and clinical medicine (11). A nomogram provides a more intuitive and understandable graphical interface which can better help clinical decisionmaking. After the correlation analysis, we first established a basic nomogram with baseline characteristics, which showed good predictive performance. Later, we combined several dynamic variation characteristics in the treatment process to further establish a response nomogram, which demonstrated a better predictive performance than the baseline nomogram. There is still a lack of effective methods for predicting the response of neoadjuvant therapy for esophageal cancer. As the largest single-center retrospective study to date, we analyzed the baseline characteristics of patients and their results of hematology tests, screened out relevant characteristics, and further developed response prediction tools. The response nomogram can help clinicians to identify the possible therapeutic effects of patients early through simple and economical methods, so as to intervene early and benefit patients.

This study is a retrospective research and thus involved some selection bias. The endpoint of this study was the preoperative imaging evaluation effect, and it is still not clear whether its imaging characteristics extracted from these computed tomography (CT) scans can ultimately be converted into survival benefit. Although our prediction model has good robustness, we used bootstrap validation for internal validation but did not apply external validation. Moreover, our research objects were Chinese people with ESCC, so the ability to generalize our results to 
other regions and races is uncertain. Future prospective multicenter studies with large sample sizes should be conducted to evaluate the predictability of the model. Peripheral blood indicators are susceptible to various factors and fluctuate considerably; nonetheless, a few trends could be observed using large samples before and after treatment. These trends and phenomena are unified with the current basic research mechanism. The findings of this study indicate that doctors should, rather than simply focusing on the side effects of neoadjuvant therapy, dynamically observe patients' blood routine indicators in their daily clinical work and holistically assess the risks and benefits of the variations of these indicators in devising a clinical strategy. In the era of immunotherapy, the combination of immune checkpoint inhibitors and chemotherapeutic drugs has once again provided hope in the treatment of tumors. Apart from the expression of various antibodies, at present, most of the predictive studies on the efficacy of immunotherapy have focused on various lines of peripheral blood cells and their interactions. In the near future, with the completion of further research and acquisition of data, it is hoped that meaningful information can be gleaned from simple blood routine results.

\section{Conclusions}

This study developed a novel nomogram with good accuracy to help clinicians evaluate and predict the neoadjuvant therapy response of patients with ESCC via readily available clinical data. Through the prediction of curative effect, better monitoring and timely intervention measures can be made in the process of neoadjuvant therapy for patients. This predictive model requires external validation and further research to be applied for the benefit of patients.

\section{Acknowledgments}

Funding: None.

\section{Footnote}

Reporting Checklist: The authors have completed the TRIPOD reporting checklist. Available at http://dx.doi. org/10.21037/atm-21-1628

Data Sharing Statement: Available at http://dx.doi. org/10.21037/atm-21-1628
Conflicts of Interest: All authors have completed the ICMJE uniform disclosure form (available at http://dx.doi. org/10.21037/atm-21-1628). The authors have no conflicts of interest to declare.

Ethical Statement: The authors are accountable for all aspects of the work in ensuring that questions related to the accuracy or integrity of any part of the work are appropriately investigated and resolved. The study was approved by an independent ethical committee review board at Guangdong Provincial People's Hospital. (No. GDREC2020253H) and written informed consent was obtained from all patients. All procedures performed in this study involving human participants were in accordance with the Declaration of Helsinki (as revised in 2013).

Open Access Statement: This is an Open Access article distributed in accordance with the Creative Commons Attribution-NonCommercial-NoDerivs 4.0 International License (CC BY-NC-ND 4.0), which permits the noncommercial replication and distribution of the article with the strict proviso that no changes or edits are made and the original work is properly cited (including links to both the formal publication through the relevant DOI and the license). See: https://creativecommons.org/licenses/by-nc-nd/4.0/.

\section{References}

1. Sung H, Ferlay J, Siegel RL, et al. Global cancer statistics 2020: GLOBOCAN estimates of incidence and mortality worldwide for 36 cancers in 185 countries. CA Cancer J Clin 2021. [Epub ahead of print].

2. Chen W, Zheng R, Baade PD, et al. Cancer statistics in China, 2015. CA Cancer J Clin 2016;66:115-32.

3. Meredith KL, Weber JM, Turaga KK, et al. Pathologic response after neoadjuvant therapy is the major determinant of survival in patients with esophageal cancer. Ann Surg Oncol 2010;17:1159-67.

4. Wen J, Luo K, Liu H, et al. MiRNA Expression Analysis of Pretreatment Biopsies Predicts the Pathological Response of Esophageal Squamous Cell Carcinomas to Neoadjuvant Chemoradiotherapy. Ann Surg 2016;263:942-8.

5. Li Y, Huang HC, Chen LQ, et al. Predictive biomarkers for response of esophageal cancer to chemo(radio)therapy: A systematic review and meta-analysis. Surg Oncol 2017;26:460-72.

6. Ménétrier-Caux C, Ray-Coquard I, Blay JY, et al. Lymphopenia in Cancer Patients and its Effects on 
Response to Immunotherapy: an opportunity for combination with Cytokines? J Immunother Cancer 2019; 7:85.

7. Treffers LW, Hiemstra IH, Kuijpers TW, et al. Neutrophils in cancer. Immunol Rev 2016;273:312-28.

8. Sun Y, Zhang L. The clinical use of pretreatment NLR, PLR, and LMR in patients with esophageal squamous cell carcinoma: evidence from a meta-analysis. Cancer Manag Res 2018;10:6167-79.

9. Eisenhauer EA, Therasse P, Bogaerts J, et al. New response evaluation criteria in solid tumours: revised RECIST guideline (version 1.1). Eur J Cancer 2009;45:228-47.

10. Rice TW, Ishwaran H, Kelsen DP, et al. Recommendations for neoadjuvant pathologic staging (ypTNM) of cancer of the esophagus and esophagogastric junction for the 8th edition AJCC/UICC staging manuals. Dis Esophagus 2016;29:906-12.

11. Iasonos A, Schrag D, Raj GV, et al. How to build and interpret a nomogram for cancer prognosis. J Clin Oncol 2008;26:1364-70.

12. Pencina MJ, D'Agostino RB. Overall C as a measure of discrimination in survival analysis: model specific population value and confidence interval estimation. Stat Med 2004;23:2109-23.

13. Chao YK, Chuang WY, Yeh CJ, et al. Anatomical distribution of residual cancer in patients with oesophageal squamous cell carcinoma who achieved clinically complete response after neoadjuvant chemoradiotherapy. Eur J Cardiothorac Surg 2018;53:201-8.

14. Xie X, Luo KJ, Hu Y, et al. Prognostic value of preoperative platelet-lymphocyte and neutrophillymphocyte ratio in patients undergoing surgery for esophageal squamous cell cancer. Dis Esophagus 2016;29:79-85.

15. Galluzzi L, Humeau J, Buque A, et al. Immunostimulation with chemotherapy in the era of immune checkpoint inhibitors. Nat Rev Clin Oncol 2020;17:725-41.

16. Klein E, Becker S, Svedmyr E, et al. Tumor infiltrating lymphocytes. Ann N Y Acad Sci 1976;276:207-16.

17. Menter DG, Kopetz S, Hawk E, et al. Platelet "first responders" in wound response, cancer, and metastasis. Cancer Metastasis Rev 2017;36:199-213.

18. Harano K, Kogawa T, Wu J, et al. Thrombocytosis as a prognostic factor in inflammatory breast cancer. Breast Cancer Res Treat 2017;166:819-32.

19. Steele M, Voutsadakis IA. Pre-treatment platelet counts as a prognostic and predictive factor in stage II and III rectal adenocarcinoma. World J Gastrointest Oncol 2017;9:42-9.

20. Qian BZ, Li J, Zhang H, et al. CCL2 recruits inflammatory monocytes to facilitate breast-tumour metastasis. Nature 2011;475:222-5.

21. Tang X. Tumor-associated macrophages as potential diagnostic and prognostic biomarkers in breast cancer. Cancer Lett 2013;332:3-10.

22. Wen J, Ye F, Huang X, et al. Prognostic Significance of Preoperative Circulating Monocyte Count in Patients With Breast Cancer: Based on a Large Cohort Study. Medicine (Baltimore) 2015;94:e2266.

23. Wilson MJ, van Haaren M, Harlaar JJ, et al. Long-term prognostic value of preoperative anemia in patients with colorectal cancer: A systematic review and meta-analysis. Surg Oncol 2017;26:96-104.

24. Grimm T, Buchner A, Schneevoigt B, et al. Impact of preoperative hemoglobin and CRP levels on cancerspecific survival in patients undergoing radical cystectomy for transitional cell carcinoma of the bladder: results of a single-center study. World J Urol 2016;34:703-8.

25. Pergialiotis V, Daskalakis G, Thomakos N, et al. Prechemotherapy Hemoglobin Levels as a Predictive Factor of Ovarian Cancer Survival: A Systematic Review and Meta-Analysis. Am J Clin Oncol 2019;42:725-31.

26. Coffelt SB, Wellenstein MD, de Visser KE. Neutrophils in cancer: neutral no more. Nat Rev Cancer 2016;16:431-46.

27. Kazandjian D, Gong Y, Keegan P, et al. Prognostic Value of the Lung Immune Prognostic Index for Patients Treated for Metastatic Non-Small Cell Lung Cancer. JAMA Oncol 2019;5:1481-5.

28. Deng HY, Wang WP, Wang YC, et al. Neoadjuvant chemoradiotherapy or chemotherapy? A comprehensive systematic review and meta-analysis of the options for neoadjuvant therapy for treating oesophageal cancer. Eur J Cardiothorac Surg 2017;51:421-31.

(English Language Editor: J. Gray)

Cite this article as: Wang S, Zhou Z, Tian D, Huang S, Wang C, Gao Z, Ben X, Tang J, Xie L, Zhou H, Zhang D, Shi R, Deng C, Zhuang W, Ding Y, Qiao G. A validated nomogram integrating hematological indicators to predict response to neoadjuvant therapy in esophageal squamous cell carcinoma patients. Ann Transl Med 2021;9(8):703. doi: 10.21037/atm-21-1628 